\title{
LA ENSEÑNARA DE LA ESCRITURA EN LA EDUCACIÓN SUPERIOR: UNA EXPERIENCIA DIDÁCTICA EN TORNO AL MACROGÉNERO "DESCRIPCión de Flujograma"
}

\author{
Teaching Writing in Higher Education: A Pedagogical Experience with the 'Flow \\ Chart Description' Macro-Genre
}

APPRENDRE L'ÉCRITURE DANS L'ENSEIGNEMENT SUPÉRIEURE : UNE EXPÉRIENCE

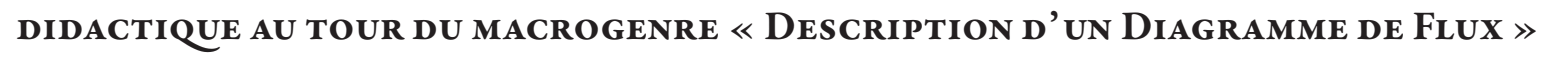

\author{
Cecilia Serpa \\ Magíster en Psicología Cognitiva y \\ Aprendizaje (UAM/Flacso). \\ Profesora adjunta regular, \\ Universidad Nacional Almirante \\ Brown, Argentina. \\ cecilia.serpa@unab.edu.ar \\ https://orcid. \\ org/0000-0002-5166-649X
}

\begin{abstract}
RESUMEN
Este artículo presenta evidencias a favor de la enseñanza de la escritura a través del currículum, siguiendo una didáctica explícita basada en el género, bajo el modelo de docente asociado. Para ello, expone una experiencia que se llevó a cabo con estudiantes del curso Herramientas Computacionales y Programación para la Ingeniería y la Ciencia, en una universidad en la Provincia de Buenos Aires, Argentina, en torno a la elaboración de un macrogénero: la Descripción de Flujograma. En este marco, se realiza una caracterización del macrogénero desde la perspectiva de la lingüística sistémico-funcional, en particular, de la Escuela de Sídney, la cual permitió delimitar con precisión el objeto de enseñanza. Luego, se exponen los fundamentos de la tarea docente. En tercer lugar, se presentan los resultados obtenidos mediante la implementación de una rúbrica o matriz de evaluación, la cual permitió ponderar el desempeño de los sujetos tanto en la producción de la primera versión del texto como en su versión editada. Por último, se argumenta a favor de las opciones teóricas, metodológicas y didácticas que sustentan la propuesta de enseñanza de la escritura que actualmente se lleva a cabo en la universidad, sin dejar de lado la revisión de los puntos que deberían ser atendidos de cara a experiencias futuras.
\end{abstract}

Palabras clave: educación superior; escritura a través del currículum; géneros profesionales; macrogénero; Descripción de Flujograma; LSF.

\section{Abstract}

This article presents evidence in favor of teaching and writing across the curriculum, following a genre-based explicit approach, and an adjunct teaching model. This is done by reporting an experience that was carried out with students enrolled in the Computer-based Tools and Programming for Engineering and

Recibido: 2020-06-25 / Aceptado: 2020-08-10 / Publicado: 2021-01-30

https://doi.org/10.17533/udea.ikala.v26n01a02 
Science course, being offered at a university in the province of Buenos Aires, Argentina. This experience had to do with developing the Flowchart Description macro-genre. Firstly, the macro-genre is characterized from the perspective of systemic functional linguistics, particularly the Sydney School, which made it possible to precisely delimit the object of teaching. Secondly, the foundations of the teaching process were presented. Thirdly, a discussion of the results obtained from the implementation of a rubric or evaluation matrix was provided, which weighed participants' performance in the production of both their first draft and their final version. Lastly, the theoretical, methodological, and didactic options supporting the writing model currently deployed at this university were shown and issues to be addressed in future experiences were discussed.

Keywords: higher education; writing across the curriculum; professional genres; macro-genres; SFL, Flowchart Description.

\section{RÉSUMÉ}

Cet article présente des éléments en faveur de l'enseignement de l'écriture dans l'enseignement supérieur tout au long des carrières, de manière transversale, en suivant une didactique explicite basée sur le genre, sous le modèle de l'enseignant associé. À cette fin, il présente une expérience qui a été réalisée avec des étudiants du cours « Outils informatiques et programmation pour l'ingénierie et la science » (Herramientas Computacionales y Programación para la Ingeniería y la Ciencia, dans une université de la province de Buenos Aires (Argentine), autour du développement d'un macrogenre : la description d'un diagramme de flux. Dans ce cadre, une caractérisation du macrogenre est faite du point de vue de la linguistique systémique-fonctionnelle, en particulier, de l'École de Sydney, qui a permis de délimiter précisément l'objet de l'enseignement. Ensuite, les fondamentaux de la tâche d'enseignement sont exposés. Troisièmement, les résultats obtenus par la mise en œuvre d'une rubrique ou d'une matrice d'évaluation sont présentés, ce qui a permis de pondérer la performance des sujets dans la production de la première version du texte et de sa version éditée. Enfin, il est plaidé en faveur des options théoriques, méthodologiques et didactiques qui soutiennent la proposition d'enseigner l'écriture qui est actuellement réalisée à l'université, sans laisser de côté l'examen des points qui devraient être abordés pour les expériences futures.

Mots-clés : enseignement supérieur ; écriture à travers le curriculum ; genres professionnels ; macrogenre ; Description d'un Diagrame de Flux ; linguistique fontionnelle systémique. 


\section{Introducción}

La literatura existente concuerda con respecto a que el encuentro entre las y los estudiantes que ingresan a la universidad y la lectura y la escritura en este ámbito constituye un escollo que merece ser atendido (Moyano, 2008), en tanto, en ocasiones, termina por atentar contra la continuidad y el egreso del nivel superior. Sin embargo, no existe consenso en torno las propuestas más ajustadas para hacer frente a estos desafíos (Hyland, 2002; Moyano, 2004). De allí que, tanto en la República Argentina como en el resto del mundo, actualmente convivan propuestas que van desde cursos o talleres que se dictan en el nivel de pregrado o en los primeros años de la carrera - generalmente orientados a "remediar" la situación de escaso desarrollo de habilidades de lectura y escritura, generado por experiencias educativas catalogadas de "deficientes" en los niveles previos, como la educación secundaria - hasta la formación en lectura y escritura dentro de las carreras y a lo largo de las asignaturas (Moyano, 2004). En el marco de este debate, Moyano (2004) sostiene que

[...] la carrera universitaria exige [...] el aprendizaje de nuevos géneros, entendidos como actividad social llevada a cabo a través del lenguaje (Martin, 1993) [...]. El desafío consiste en diseñar un programa para el acceso gradual de los alumnos que hoy llegan a las universidades a los géneros académicos (p. 4).

En línea con este debate, la Universidad Nacional Guillermo Brown (UNaB), en Argentina, cuenta actualmente con la Subárea Competencias en Discurso Profesional y Académico, cuyo objetivo es que los y las estudiantes mejoren la comprensión lectora de textos especializados y desarrollen competencias de escritura, con la finalidad, como señala Halliday (2004), de aprender a través del lenguaje. Por ello, la subárea se despliega transversalmente a lo largo de las carreras y trabaja mediante una didáctica explícita basada en el género (Moyano, 2007; 2013), bajo el modelo de "docente asociado", esto es, docentes especialistas en discurso y escritura, que se asocian con los docentes de las asignaturas, a fin de acordar la enseñanza de determinado género, establecer tiempos y espacios para hacerlo, etc. (Moyano, 2018, p. 243).

La oferta académica de la UNaB incluye la “Tecnicatura Universitaria en Automatización y Control" (AyC), una carrera de pregrado con una duración de tres años. En este marco, se propone "formar profesionales que posean las capacidades requeridas para el diseño, mantenimiento y operación" de tecnologías de automatización y control en diversas actividades industriales y productivas, "cuyo carácter transversal a los distintos sectores de actividad productiva" se ajusta a la diversificación del parque industrial localizado en la región de influencia. En este sentido, se espera que el egresado o egresada se desempeñe en "tareas de diseño, mantenimiento", operación y puesta a punto de "máquinas automáticas, controles de procesos automatizados y mecanismos robóticos”, entre otras cuestiones de orden técnico (UNaB, s. f.).

La asignatura "Herramientas Computacionales y Programación para la Ingeniería y la Ciencia” (HCyP), de dicha carrera, es el primer espacio de formación específica con que cuentan las y los estudiantes. Allí tienen su primer contacto con algunas herramientas computacionales y comienzan a conocer los rudimentos de la programación. Además, en este espacio se encuentran por primera vez con docentes de la subárea, por lo que comienzan a reflexionar, paralelamente, sobre los textos y la escritura, siempre en el marco de los géneros propios del campo disciplinar.

En este contexto, durante el segundo semestre de 2019, se llevó a cabo un proceso de investigación y docencia en torno a la elaboración de un macrogénero, Descripción de Flujograma (DF), cuyos resultados se discuten en este trabajo. Más precisamente, en este artículo se realiza, en primer lugar, una caracterización del macrogénero (Eggins, 2004; Eggins y Martin, 2003; Martin y Rose, 2008) desde la perspectiva de la lingüística sistémico-funcional (LSF) (Halliday y Matthiessen, 2004; Martin y Rose, 2007, 2008); en segundo lugar, se explica brevemente de qué forma se efectuó la tarea 
de enseñanza; en tercer lugar, se presentan los resultados obtenidos; y, por último, se argumenta a favor de la didáctica basada en el género, en el marco de las asignaturas de la educación superior, sin dejar de lado la revisión de los puntos que deberían ser atendidos de cara a experiencias futuras.

\section{Marco teórico}

En este apartado se definen los géneros científicos (Martin y Rose, 2008), con especial énfasis en los informes y las explicaciones. Luego, se caracteriza al macrogénero DF, una construcción original que orientó el diseño de las clases en las que se llevó a cabo la experiencia de enseñanza, objeto de análisis de este trabajo.

\section{Los géneros científicos}

El concepto de género es entendido en este trabajo como una combinación de significados correspondientes a las variables de campo, tenor y modo, en tanto estas se relacionan de forma sistemática con determinados procesos sociales (Martin, 1992). En términos de Martin y Rose (2008), tal integración o configuración de significados está asociada al propósito o función social de un texto como práctica social en una cultura dada.

En este sentido, el género supone una organización estructural o estructura esquemática determinada, organizada como una serie de etapas o pasos, y una combinación específica de los significados correspondientes a las variables del registro. De allí que los autores planteen la propuesta de

[...] modelar el género en el estrato de la cultura, más allá del registro, donde podía funcionar como un patrón de patrones de campo, tenor y modo. En este movimiento, hemos remodelado el lenguaje en contexto social como un sistema semiótico, en el que "situación" y "cultura" se reconstruyen como estratos semióticos sociales: el registro y el género (Martin y Rose, 2008, p. 16). ${ }^{1}$

1 Las traducciones son, en todos los casos, de la autora de este trabajo.
En este marco, el campo científico posee formas específicas de semiotizar el mundo natural, que se traducen en géneros propios de esta esfera. En efecto, Martin y Rose (2008) consideran que

[...] la ciencia semiotiza el mundo natural mediante generalizaciones sobre las cosas y los procesos de cuatro maneras regulares: mediante la clasificación y descripción de fenómenos, a través de la explicación de cómo suceden los procesos, indicando cómo observar fenómenos (por ejemplo, en experimentos) e informando e interpretando lo que fue observado (p. 141).

De la cita precedente se desprende la existencia de un conjunto de géneros propios del campo científico: los informes, que clasifican y describen; las explicaciones, que establecen relaciones de causa y consecuencia; los instructivos de observación y experimentación, y los informes de procedimiento, que reportan observaciones y experiencias.

De estos cuatro géneros elementales, el único que no sigue una organización temporal es el informe. En las explicaciones, esta temporalidad está ligada al proceso que se explica, es decir, es intrínseca de las actividades a las que se hace referencia; mientras que en instructivos e informes de procedimiento, la temporalidad está ligada a lo que el lector deberá hacer, en el primer caso, y lo que el autor ya ha realizado, en el segundo. Así, "las explicaciones construyen secuencias de actividades, mientras que los informes hacen foco en las entidades, organizadas mediante clasificación y composición, más que desenvolviéndose en el tiempo" (Martin y Rose, 2008, p. 166).

En el caso particular del macrogénero DF, este combina los géneros informe descriptivo, informe composicional y explicación secuencial. La relación entre estos géneros de carácter científico en el macrogénero DF resulta pertinente y lógica. En efecto, Martin y Rose (2008) consideran que, más allá de sus diferencias, informes y explicaciones comparten un conjunto de recursos lingüísticos, dado que en ambos casos se construyen relaciones entre fenómenos. 
Tabla 1 El informe y la explicación, y sus tipos, propósitos y etapas

\begin{tabular}{|c|c|c|c|}
\hline Género & Tipo & Propósito & Etapas* \\
\hline \multirow[t]{3}{*}{ Informe } & Descriptivo & Clasificar un fenómeno y describir sus rasgos & Clasificación ^ Descripción \\
\hline & Taxonómico & $\begin{array}{l}\text { Subclasificar un conjunto de fenómenos, en función de una clase } \\
\text { general }\end{array}$ & Clasificación ^ Tipo \\
\hline & Composicional & Describir los componentes de una entidad & Clasificación ${ }^{\wedge}$ Componentes \\
\hline \multirow[t]{4}{*}{ Explicación } & Secuencial & $\begin{array}{l}\text { Explicar un proceso con base en un encadenamiento simple de } \\
\text { causas y efectos }\end{array}$ & Fenómeno ${ }^{\wedge}$ Explicación \\
\hline & Factorial & Explicar un proceso con base en múltiples causas & \\
\hline & Consecuencial & $\begin{array}{l}\text { Explicar un proceso con base en una causa y múltiples } \\
\text { consecuencias }\end{array}$ & \\
\hline & Condicional & $\begin{array}{l}\text { Explicar un proceso con base en una causa, que puede variar } \\
\text { dependiendo de condiciones }\end{array}$ & \\
\hline
\end{tabular}

* Se usa el símbolo ^ para representar la secuencia de realización típica.

\section{El informe y la explicación}

Informes y explicaciones presentan distintas variantes, dependiendo del propósito que realizan (Martin y Rose, 2008). El género informe posee tres tipos, y la explicación, cuatro, como se sintetiza en la Tabla 1.

En relación con los informes, en todos los casos se trata de describir una entidad o fenómeno a partir de sus rasgos o características, o en función de sus componentes; o bien mediante su taxonomización con base en determinado criterio.

Cabe señalar que la etapa Clasificación se puede realizar por medio de una definición del fenómeno objeto del informe, y que, en los casos en que este género posee una segunda etapa, esta puede operar recursivamente. Por ejemplo, a fin de presentar distintas características del fenómeno que se está describiendo, se incluyen nuevas fases descriptivas.

En cuanto a las explicaciones, todos los tipos emplean secuencias de causas y efectos que encadenan los procesos entre sí. Martin y Rose (2008) denominan "secuencia de implicación" a esta clase de patrones lógicos. En este sentido, la estructura esquemática de la explicación tiene siempre dos etapas: Fenómeno ${ }^{\wedge}$ Explicación. En la primera, se indica cuál será el fenómeno explicado ${ }^{2}$ y, a continuación, se inserta la secuencia de implicaciones que lo explica. La clase de secuencia inserta es lo que determina el tipo de explicación de que se trata. En otras palabras, las explicaciones varían considerablemente en función del fenómeno que está siendo explicado, pero en todos los casos poseen un alto nivel de tecnicalidad, es decir, un importante volumen de lenguaje técnico o de especialidad, y la relación entre los eventos de una secuencia explicativa es necesariamente temporal, dado que se establecen relaciones lógicas de sucesión o simultaneidad entre ellos.

\section{Macrogénero: la Descripción de un Flujograma en el campo de la programación}

Informes y explicaciones, como se indicó más arriba, constituyen géneros elementales. Sin embargo, la teoría advierte que, en ciertos casos, los textos se organizan en términos del macrogénero (Eggins, 2004; Eggins y Martin, 2003; Martin y Rose, 2008), esto es, configuraciones de géneros elementales que realizan un único propósito social, de forma conjunta: en palabras de los autores,

2 Además de indicar el fenómeno que se explica, las explicaciones pueden comenzar o finalizar con una definición técnica de este. 


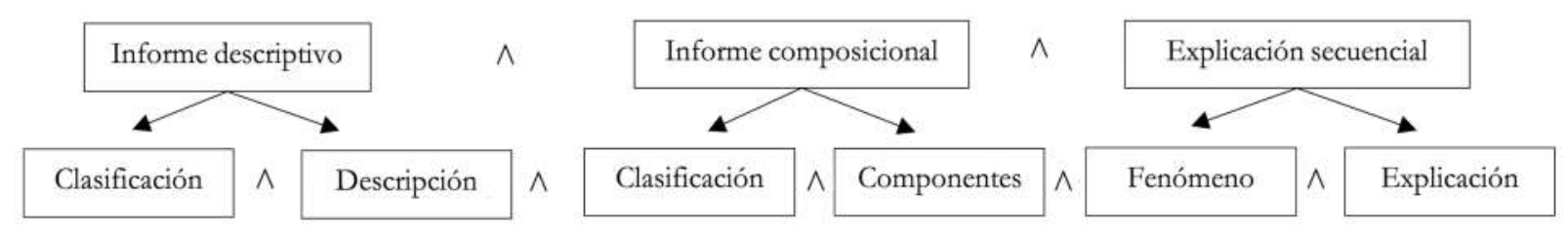

Figura 1 Estructura esquemática del macrogénero "Descripción de Flujograma"

"un texto que está formado por varios géneros diferentes” (Eggins y Martin, 2003, p. 14).

De esta manera, a continuación, se caracteriza la DF en tanto macrogénero, como la hemos descrito para los fines didácticos de la tarea de enseñanza. Para ello, se presenta la conformación de la macroestructura y las relaciones entre los géneros dentro de esta, y se exponen sus principales rasgos semántico-discursivos y léxico-gramaticales.

\section{Macroestructura}

El macrogénero DF está conformado por el informe cación. Estos constituyen textos adyacentes, que conforman un texto complejo, en analogía con los complejos clausulares:

\section{Descripción de Flujograma: Informe Descriptivo ${ }^{\wedge}$ Informe Composicional ${ }^{\wedge}$ Explicación Secuencial.}

A su vez, cada género elemental se descompone, como adelantáramos, en etapas o pasos, como muestra la Figura 1.

En este sentido, y teniendo en cuenta que lógicamente la Clasificación no se reitera en la segunda etapa, al comienzo del informe composicional, la estructura del macrogénero se puede sintetizar del siguiente modo:

\section{Descripción de Flujograma: Clasificación ${ }^{\wedge}$ Descripción ${ }^{\wedge}$ Composición ${ }^{\wedge}$ Fenómeno ${ }^{\wedge}$ Explicación.}

Ahora bien, respecto de los tipos de relaciones que establecen estos textos, es importante recordar que Martin (1994) y Martin y Rose (2008) proponen una serie de categorías para describir cómo se combinan o relacionan entre sí los

géneros elementales dentro de los macrogéneros. En particular, los autores encuentran que, así como propone la LSF para describir las relaciones entre cláusulas (Halliday y Matthiessen, 2004), los géneros pueden establecer relaciones de expansión o de proyección en el marco de un macrogénero. Entre las relaciones de expansión, se encuentran las de elaboración $(=)$, extensión $(+)$ y realce $(\mathrm{x})$. En palabras de Martin (1994),

Con la expansión, una cláusula expande otra elaborándola, extendiéndola o realzándola. Siguiendo a Halliday 1985a: 196-197, la elaboración supone replantear en otras palabras, especificar con mayor detalle, comentar o ejemplificar; la extensión involucra agregar algún elemento nuevo, presentar una excepción $\mathrm{u}$ ofrecer una alternativa; el realce supone enriquecer mediante características circunstanciales de tiempo, lugar, causa o condición (p. 62).

En el caso que nos ocupa, la relación entre los primeros dos géneros elementales que conforman este macrogénero es de expansión: extensión, dado que el informe composicional especifica o amplía el informe descriptivo. La explicación, por otra parte, establece una relación de expansión: elaboración con los dos primeros géneros, pues la explicación secuencial ejemplifica el fenómeno previamente descripto. Siguiendo el sistema notacional que utiliza la LSF, el macrogénero se describe, entonces, del siguiente modo:

$$
\begin{gathered}
\text { Descripción de Flujograma: Informe Descriptivo + Informe } \\
\text { Composicional = Explicación Secuencial. }
\end{gathered}
$$

La Figura 2 muestra esta relación entre los géneros y sus etapas o pasos.

En términos más concretos, este macrogénero comienza con una definición del concepto de 


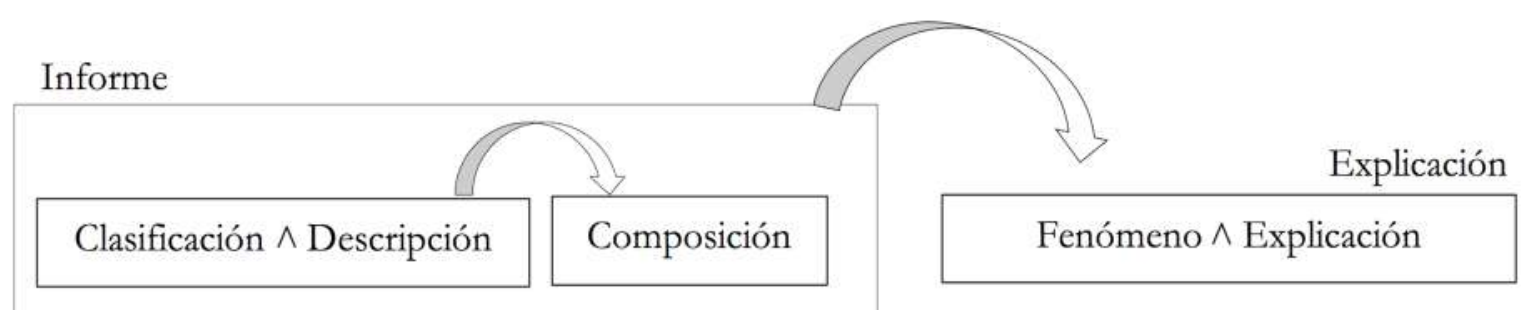

Figura 2 Relación entre los géneros que componen el macrogénero "descripción de flujograma”

flujograma y una breve caracterización de esta entidad. Esto corresponde a las etapas clasificación y descripción. Luego, la fase composición incluye un informe composicional de los elementos que conforman un flujograma. Estos elementos son los bloques del diagrama de flujo: formas geométricas como paralelogramo o rombo, utilizadas en el campo de la programación para representar acciones en un proceso o relaciones lógicas entre los elementos involucrados; por ejemplo, inicio, toma de decisión, $y$. En esta etapa, el informe composicional verbal incluye una descripción composicional visual, mediante la ilustración de los tipos de bloques, a título estrictamente complementario, dado que las palabras por sí mismas podrían bastar para describir estos elementos. Como se indica, la inclusión de los bloques de programación en el texto tiene una función estrictamente complementaria e ilustrativa en este macrogénero, es decir, no cambian ni amplía el sentido ni aporta información no dicha verbalmente. No obstante, el estudio pormenorizado de los aspectos multimodales pueden ser objeto de trabajo en futuras investigaciones.

Luego, la Explicación Secuencial incluye las dos etapas: en la etapa Fenómeno presenta un caso específico - un algoritmo dado- y en la fase Explicación explica el proceso allí representado. En este macrogénero, el flujograma se coloca como complemento de la explicación verbal, para ilustrar el proceso y facilitar la comprensión; y la explicación avanza a partir de un eje simultáneamente temporal - el flujo pasa de un bloque al siguiente - y causal — cada bloque es consecuencia de la acción realizada en el bloque previo-.
Por lo tanto, es posible afirmar que, como se puede deducir de la caracterización enunciada, el propósito de este macrogénero, la DF, es Describir un Flujograma dado, en tanto entidad del campo de la programación.

\section{Algunos recursos semántico-discursivos y léxico-gramaticales}

El conjunto de recursos semántico-discursivos y léxico-gramaticales de este macrogénero varía en función de los géneros que lo componen.

En relación con los Informes, desde el punto de vista del sistema de IDEACIÓN, se observa un predominio de figuras relacionales, organizadas en torno a procesos, realizados por verbos; y participantes, realizados por grupos nominales o preposicionales, como evidencian los siguientes ejemplos:

(1) Un flujograma es la representación gráfica de un algoritmo.

(2) El diagrama de flujo incluye diversos símbolos estandarizados para cada función.

(3) El rectángulo representa la realización de una operación o actividad relativas a un procedimiento.

Las cláusulas relacionales sirven para caracterizar e identificar (Eggins, 2004). Respecto a su composición léxico-gramatical, la mayor parte de las cláusulas relacionales realizan su proceso mediante un verbo relacional — ser, estar, tener-, cargan con dos participantes inherentes y siguen la estructura xes/tiene $y$.

Los participantes se pueden realizar mediante un grupo nominal inespecífico (indefinido) o 
específico (definido) o una frase preposicional. En muchos casos, además, estos participantes son abstracciones o incluyen una metáfora gramatical ideativa: representación, flujo, decisión son algunos de los condensados semánticos que realizan la función de participante, en tanto se trata de nominalizaciones, es decir, "empaquetamientos de secuencias de actividades" (Martin y Rose, 2007, p. 106). En estos casos, las actividades son codificadas "como si ellas fueran cosas", por lo que "los procesos son realizados por una clase gramatical atípica, como un nombre" (p. 106). Esto se puede observar en el segundo participante de los ejemplos (1) y (3) más arriba, y en los ejemplos (4) y (5), a continuación:

(4) Un flujograma es una representación visual de un proceso o algoritmo determinado.

(5) El flujo continúa del mismo modo.

En términos semánticos, es posible identificar qué tipo de experiencia construyen las cláusulas organizadas en torno a procesos relacionales: interna o externa

84 (Martin y Rose, 2007), la realidad se representa como 'siendo', más que como 'haciendo' (materiales) o como 'percibiendo' (mentales). En relación con esto, en el caso específico del macrogénero que estamos analizando, la experiencia representada corresponde al mundo externo, dado que toma por objeto la descripción del flujograma.

Las cláusulas relacionales se agrupan en dos grandes modelos, que, a su vez, poseen algunas variantes internas: por una parte, existen las cláusulas relacionales "atributivas". En estos casos, una entidad se clasifica como miembro de una clase; se la caracteriza: $x$ tiene la caracteristica y o $x$ pertenece a la clase $y$, como en "Un flujograma es una forma de representación gráfica”. Por otra, la teoría reconoce un segundo tipo de relacionales, llamadas "identificatorias". Las cláusulas de esta clase identifican determinadas entidades y siguen la fórmula $x$ es igual a $y$, como en "Un flujograma es la representación gráfica de un algoritmo”. La LSF afirma que, al menos para el inglés, las cláusulas de identificación contrastan con las de atribución, por el hecho de que las primeras son reversibles, pero no las segundas (Eggins, 2004; Halliday y Matthiessen, 2004).

Las cláusulas atributivas e identificatorias pueden ser, a su vez, intensivas, posesivas o circunstanciales. En el primer caso, es decir, en las cláusulas intensivas, se ofrece una caracterización por rasgos, como en el ejemplo (2) más arriba y en el (6) a continuación; en cambio, si la cláusula es posesiva, se orienta a la identificación por posesión, como en el ejemplo (7); finalmente, en el caso de las cláusulas circunstanciales, la identificación de un elemento se realiza a partir de su ubicación en el tiempo, el espacio u otra circunstancia, como en el ejemplo (8).

(6) Un flujograma está formado por un conjunto de bloques.

(7) El flujograma tiene bloques que representan acciones del proceso.

(8) Los bloques del flujograma se ubican formando una línea vertical.

En el caso del macrogénero que estamos analizando, la función descriptiva y composicional se realiza, además, mediante la identificación de la función o acción que realiza cada bloque, como iniciar o indicar en los ejemplos que siguen:

(9) El rectángulo de puntas redondeadas inicia el proceso.

(10) El bloque Entrada por teclado indica que el usuario debe ingresar datos mediante el teclado.

En síntesis, el informe descriptivo y composicional se realiza mediante cláusulas relacionales atributivas e identificativas de tipo intensivo, posesivo y circunstancial. La metáfora gramatical ideativa puede funcionar como participante y las cláusulas se vinculan por medio de recursos del sistema de CONJUNCIÓN o metáforas lógicas (Martin y Rose, 2008); principalmente, a través del uso de conjunciones internas y externas aditivas, como además, similarmente o como.

Por su parte, la Explicación Secuencial incorpora dos elementos clave que transforman completamente la manera en que se representa la experiencia: el tiempo 
y las relaciones de causalidad y consecuencialidad. En este caso, se conjugan figuras relacionales con figuras materiales, dado que no solo se deben definir o describir los elementos que conforman el flujograma a medida que se explica el proceso, sino, además, indicar qué acciones suceden a medida que avanza el flujo, como en los ejemplos (11), (12) y (13):

(11) El quinto es un bloque de decisión y este realiza la pregunta ' $\mathrm{i} \mathrm{A}=\mathrm{B}$ ?'.

(12) Entonces, el flujo se conduce ahora hacia el bloque octavo, muestra en la pantalla o imprime "B es el mayor" y el proceso finaliza en el bloque Fin.

(13) Luego, el algoritmo entra en un bloque de decisión lógica, y este pregunta si "A" y "B" son iguales. Si la respuesta es afirmativa, vuelve a solicitar el ingreso de datos tal y como lo indica la línea de flujo.

En este sentido, se incorporan procesos como servir, realizar, solicitar, evaluar, entre otros, y los participantes a ellos asociados. Se trata de procesos propios del campo de la programación, que aluden a las acciones que prototípicamente realiza un algoritmo en cada uno de sus pasos. Si bien muchos de ellos tienen un matiz mental, como el verbal solicitar o el cognitivo evaluar, en todos los casos poseen la función de indicar qué acción está realizando el algoritmo. Es decir que, en tales casos, los procesos están siendo utilizados en un sentido metafórico, en tanto se les asignan atributos humanos a los bloques del Flujograma (de allí que "decida", "solicite", etc.).

En cuanto a la flexión, cabe señalar que la especificidad del macrogénero conduce a que todos los verbos aparezcan conjugados en presente, dado que el propósito descriptivo resulta dominante. En consecuencia, las relaciones de temporalidad y causalidad de la explicación que no se explicitan mediante la flexión verbal se establecen por medio de recursos del sistema de CONJUNCIÓN - conjunciones de tiempo, causa y condición: si, entonces, por lo tanto, luego, etc. - o mediante "metáforas lógicas que realizan el mismo significado a través de recursos de otros sistemas" - por ejemplo: en caso de, esto se debe a, lleva a la consecuencia de, etc.- (Martin y Rose, 2007, p. 150).
Un elemento por destacar es la forma en que se describen las acciones dependientes de los bloques If o de Toma de Decisión. Estos introducen un sistema binario de opciones para el flujo a partir de una pregunta: tienen una entrada y dos salidas posibles excluyentes, que bifurcan el recorrido. Si la respuesta a la pregunta que introduce el bloque del programa es sí, el flujo sigue un camino - generalmente, hacia abajo o hacia la derecha-. Si la respuesta del programa es no, el proceso sigue en otra dirección —en general, hacia la izquierda, en términos de la representación visual-. Entonces, dado que se trata de instancias en las que el programa se ramifica o bifurca, presenta una dificultad especial para su descripción, dado que debe expresar el carácter condicional de cada uno de los cursos posibles y marcar claramente la oposición excluyente entre estas opciones. En esta instancia, por tanto, resulta clave el uso de los recursos del sistema de CONJUNCIÓN, que construyen significado condicional y opositivo, como se puede ver en el ejemplo (13) más arriba. La flexión verbal de futuro puede complementar esta clase de significados.

En síntesis, la etapa Fenómeno de la Explicación Secuencial se realiza con una cláusula relacional que indica qué representa el diagrama que funciona como ejemplo — "Este Flujograma representa Z”- mediante una figura organizada en torno a procesos como representar, corresponder, graficar-; y la etapa Explicación se efectúa por medio de cláusulas relacionales y materiales, conectadas con recursos de conjunción y metáforas lógicas que establecen relaciones de temporalidad y causalidad, principalmente, y de condicionalidad y oposición, de ser necesario.

Por otra parte, en relación con la metafunción interpersonal, cabe observar que el macrogénero rechaza cualquier recurso lingüístico o discursivo que evidencie la relación entre los participantes del evento. Esto significa que las huellas de subjetividad están borradas: el discurso es monologal —en términos del sistema de COMPROMISO- y las valoraciones están ausentes -en términos del 
sistema de ACTITUD- (Hood y Martin, 2005; Martin, 2000a, 2000b; Martin y White, 2005).

\section{De la caracterización del macrogénero a su enseñanza}

Las intervenciones docentes se realizaron siguiendo el modelo didáctico de Moyano (2007; 2013). Esta propuesta de enseñanza constituye una adaptación de la de la Escuela de Sídney (Martin, 1999; Rose y Martin, 2012), se organiza en torno al concepto de género (Martin y Rose, 2008), supone una didáctica explícita (Bernstein, 1990) y parte de la convicción de que el aprendizaje se da en un proceso interactivo de construcción. Más aún, durante la enseñanza, el o la docente lleva a cabo un fuerte andamiaje (Rose y Martin, 2012; Wood et al., 1976), dado que despliega los apoyos o guías para que los y las estudiantes avancen en este proceso constructivo.

Una particularidad de este modelo (Moyano, 2007) es su reconceptualización de la categoría de andamiaje, dado que aquí se realiza un especial énfasis en el diálogo como forma privilegiada para sostener el aprendizaje: "Para nosotros la metáfora del andamiaje no refuerza lo suficiente el hecho de que esa orientación tiene lugar a través del despliegue diálogo" (Rose y Martin, 2012, p. 55). En efecto, el modelo didáctico defiende la orientación mediante la interacción en el contexto de la experiencia compartida, como forma privilegiada de andamiaje (Martin, 1999; Moyano, 2011).

En concreto, la propuesta didáctica prevé distintas instancias de trabajo: deconstrucción del género, diseño y construcción de ejemplares genéricos, y edición de los escritos, como se observa en la Figura 3.

En cuanto al primer paso, se trata de la lectura colectiva de un ejemplar modélico del género o macrogénero para realizar su deconstrucción. Esta tarea se lleva a cabo con el grupo clase y supone la mediación de la o el docente, a fin de que los y las estudiantes - a través de la interacción, el debate y la sistematización - identifiquen el propósito social del género o macrogénero, su estructura esquemática y los recursos semántico-discursivos y léxico-gramaticales prototípicos.

El hecho de que este modelo comience con la tarea de deconstrucción implica que el aprendizaje de

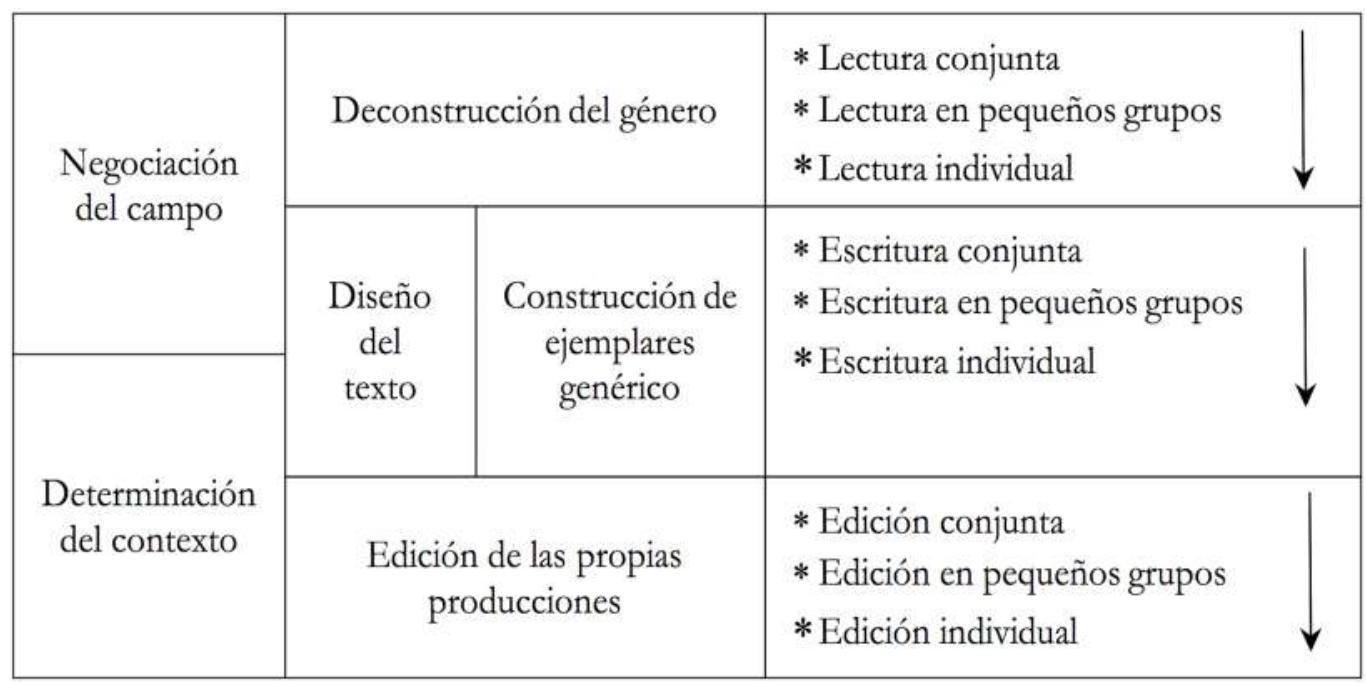

Figura 3 Propuesta didáctica

Fuente: Moyano (2007). 
la escritura se efectúa a partir de la lectura. Dicho de otro modo, se trata de un modelo ideado para la apropiación de la escritura, que toma la deconstrucción del género a ser enseñado como primer paso, lo que supone, por lo tanto, la enseñanza también de unas formas específicas de lectura.

La relevancia de esta decisión didáctica se pone de manifiesto si se tiene en cuenta que los y las estudiantes que participan sistemáticamente de instancias didácticas en las que se utiliza este modelo, construyen - a mediano y largo plazo- estrategias de lectura que les permiten enfrentar la producción autónoma de géneros o macrogéneros novedosos, tanto en el ámbito académico como en el profesional.

Cabe señalar que, durante la deconstrucción conjunta de la experiencia que estamos reportando, se fueron extrayendo y sistematizando los recursos que los y las estudiante luego "tendrían a mano" a la hora de escribir. En esta línea, se generaron esquemas y se sistematizaron figuras, estructuras clausulares y elementos léxicos. Entre estos últimos, se incluyeron tanto las metáforas gramaticales ideacionales y lógicas, como las conjunciones y el léxico específico del campo disciplinar (generalmente, abstracciones). También se analizaron las relaciones nucleares entre los elementos que conforman la cláusula y se reflexionó sobre su organización.

Todos estos elementos, sistematizados en tablas y esquemas, pasaron a formar parte de los insumos de escritura. Además, durante esta instancia se hizo especial énfasis en la centralidad del proceso y en los participantes semánticamente más inherentes, y se observó la colocación periférica del resto de los elementos, como las circunstancias y las conjunciones (Martin y Rose, 2007).

El modelo (Moyano, 2007) avanza con las tareas de diseño y construcción, que se pueden realizar con el grupo clase, en pequeños grupos, en parejas o de forma individual, dependiendo de las necesidades específicas de los y las estudiantes, del texto, de la consigna disparadora, etc.
El diseño es una subetapa de la construcción, por lo que una y otra instancia suponen tareas diversas y centrales para la apropiación del proceso de escritura. En este sentido, idealmente, diseño y construcción deberían ser abordadas en distintos momentos - sean clases o intervenciones-; sin embargo, ocasionalmente, por cuestiones de negociación entre pares (Moyano y Giudice, 2016), se realizan de forma integrada, como en el caso que comentamos aquí.

En concreto, el diseño del texto tiene como propósito realizar una primera esquematización del trabajo a producir: la identificación de qué se va a decir, cómo, por qué, etc.; es decir, se trata de una instancia de planificación del propio trabajo, tomando como base la información obtenida durante la deconstrucción. Luego, a partir sus diseños, los y las estudiantes proceden a la construcción del texto, es decir, a la primera producción completa. En estas instancias, el docente orienta la reflexión en torno a las decisiones que deben tomar los autores, y ofrece criterios para ponderar la pertinencia de tales decisiones.

Sobre la base de estas primeras producciones se lleva a cabo la última instancia de intervención: la edición conjunta, que viene seguida de la edición en pequeños grupos o individual. En este momento del proceso se selecciona al menos uno de los textos producidos por los y las estudiantes, y se lee de forma conjunta, con el grupo clase, a fin de poner de manifiesto las producciones totales o parciales que se ajustan al texto modelo deconstruido al inicio y las que necesitan edición, para aproximarse a este en mayor grado.

En esta etapa, la figura del docente nuevamente resulta central, dado que oficia de autor experto, orienta la mirada de los y las estudiantes hacia los aspectos del texto que deben ser atendidos, $\mathrm{y}$ ofrece caminos alternativos para solucionar los eventuales problemas.

El modelo didáctico, por lo tanto, desalienta la devolución individual de los borradores bajo 
sistemas de comentarios, y alienta, por el contrario, el debate colectivo en torno a formas alternativas de resolución de los problemas que enfrenta quien escribe. De este modo, no solo se intervienen las producciones concretas de los y las estudiantes, sobre la escritura en proceso, orientando su reescritura, sino que, además, se hacen explícitas la formas de hacer con el texto que lleva a cabo a un escritor que sabe más (Rogoff, 1994; Vigotsky, 1988; Wood et al., 1976).

En el caso de esta experiencia, durante la edición conjunta se retomaron los insumos producidos en la etapa de deconstrucción - tablas y esquemas que sistematizaban lascaracterísticas del macrogénero-. Es decir, la reflexión y la sistematización de las características del macrogénero se realizaron durante la deconstrucción - sobre la base de un texto modelo- y, posteriormente, durante la edición con base en sus propios textos en proceso-.

Es importante destacar que, en la edición conjunta, se reforzaron tres cuestiones que aparecieron como recurrentes: la dificultad de definir una entidad de un campo específico, en este caso, el flujograma; los desafíos que supone describir un proceso que incluye condicionalidad, representada en este caso por los bloques If o de Toma de Decisión; y la aparente influencia que ejerció un trabajo realizado previamente (Serpa, 2019), sobre otro género en la primera construcción individual de este macrogénero. Discutiremos estos temas al analizar los resultados.

Finalmente, cabe señalar que, en la instancia de edición, se ofrece a los y las estudiantes una versión ajustada de la rúbrica de evaluación (Norton y Wiburg, 2003) diseñada ad hoc para andamiar la escritura. Este recurso les explicita los criterios para la autoedición de sus escritos, los mismos criterios sobre los que se reflexionó en la clase de edición.

En síntesis, el modelo didáctico que seguimos supone las etapas de deconstrucción, diseño, construcción y edición; en el proceso de enseñanza, se establecen de manera alternativa momentos de producción y trabajo entre todos, en pequeños grupos e individuales. Paralelamente, el modelo no solo se ocupa de la enseñanza de la escritura, sino también de la lectura - como afirmamos más arriba- y de contenidos disciplinares, mediados por la lectura y la escritura.

En este sentido, la enseñanza del macrogénero DF se llevó a cabo teniendo en cuenta la caracterización presentada en el apartado precedente, es decir, utilizando las categorías que ofrece la LSF. Principalmente, se enfocó en el propósito del texto, en su macroestructura y en los recursos semántico-discursivos y léxico-gramaticales.

\section{Método}

A continuación se presentan los datos recogidos en cada comisión o grupo de sujetos, según estudiante y según dimensión de análisis del texto. También se describe el tipo de análisis realizado para obtener las dimensiones del macrogénero.

\section{Datos recogidos}

En relación con la producción de los datos, el modelo didáctico presentado más arriba supone, como se adelantó, el diseño de una rúbrica de evaluación que no solo funciona como apoyo durante el proceso, en tanto se ofrece una versión adaptada de este insumo a los y las estudiantes, sino también como herramienta para la evaluación de resultados en la enseñanza y el aprendizaje de cada género o macrogénero.

En particular, las rúbricas se diseñan ad hoc para cada género o macrogénero, en función de las dimensiones o los criterios del texto a los que se haya atendido durante la deconstrucción. Estos insumos operan en dos sentidos: por una parte, permiten valorar y calificar el desempeño de los y las estudiantes, y garantizan la evaluación estricta de aquello que haya sido efectivamente enseñado; por otra, constituyen una herramienta de control para la enseñanza, en tanto ofrecen evidencias del curso general del proceso de trabajo en el aula y de los resultados alcanzados por el grupo. 
En este caso específico, en relación con las dimensiones de análisis y los aspectos en los que se enfocó durante las clases, se decidió agrupar los rasgos del macrogénero en estructura esquemática (inclusión de los tres géneros elementales en el orden previsto, desarrollados de manera exhaustiva y claramente diferenciados), tipo de lenguaje (tecnicalidad, uso de cláusulas relacionales y materiales en los casos en que corresponde, metáfora gramatical ideacional, conjunciones y metáfora gramatical lógica), recursos gramaticales (relaciones nucleares, flexión verbal) y recursos gráficos y normativos (adecuación a las condiciones formales de entrega, como tipo y tamaño de fuente, entre otras; y adecuación normativa, como distinción de mayúsculas y minúsculas, uso del tildes, etc.). Las rúbricas contaron con descriptores cualitativos para cada nivel de desempeño, organizado en una gradación de cinco niveles.

Por último, respecto a la cantidad de sujetos, inicialmente las comisiones (C) contaron con 14 (C1) y 22 (C2) estudiantes. De estos, 7 de la C1 y 8 de la C2 entregaron el borrador; y 8 y 9 escribieron la versión final, respectivamente. Cabe señalar que constituyen el $100 \%$ de los sujetos que completaron la asignatura y la regularizaron.

Cabe señalar que, dado que la matriz de evaluación constituye un insumo simultáneamente para la tarea de docencia y de investigación, fue administrada por la misma persona, es decir, la autora de este trabajo.

\section{Análisis de los datos}

Para la producción y el análisis de los datos en el marco de este trabajo, cada dimensión se subdividió en el conjunto de rasgos o criterios que conforman el macrogénero, se asignó un valor máximo total de 10 puntos por dimensión y se distribuyeron esos 10 puntos máximos entre los distintos rasgos o niveles. De este modo, cada dimensión podía recibir hasta 10 puntos máximo. Luego, se hizo el promedio entre las cuatro dimensiones del macrogénero.
Con base en estos criterios, el desempeño de cada estudiante fue evaluado en dos momentos del proceso: en la primera entrega, después de la clase de diseño y construcción; y en la entrega final, tras la clase de edición conjunta.

\section{Resultados}

A continuación se presentan los resultados obtenidos en cada comisión o grupo de sujetos, a partir de la evaluación de los borradores (o primera construcción del texto) y las versiones finales (o texto editado), mencionados en las figuras como "Borrador" y "Final", respectivamente. Luego, se exponen los resultados, en los que se analiza separadamente cada dimensión evaluada mediante la rúbrica.

\section{Grado de avance entre borrador y versión final}

La Figura 4 permite observar las diferencias entre la primera construcción del texto y la versión editada para ambos grupos de estudiantes. En efecto, todos los participantes mostraron un crecimiento relativo entre ambas instancias: en promedio, las producciones avanzaron en 1,62 puntos (sobre 10) entre las dos versiones. La C1 creció en 2,69 y la C2, en 0,55 .

Pese al escaso margen de avance que evidencian los sujetos de la C2, mostraron un desempeño más

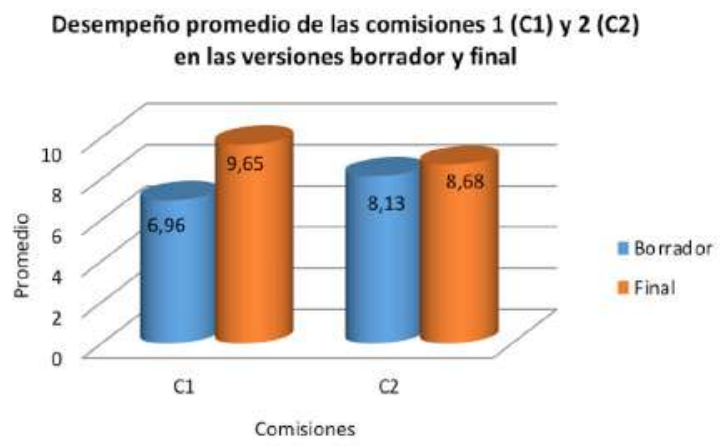

Figura 4 Desempeño promedio de las comisiones 1 (C1) y 2 (C2) en las versiones borrador y final 
ajustado en el borrador, respecto de los borradores producidos por los y las estudiantes de la $\mathrm{C} 1$. Es decir, obtuvieron en promedio resultados más altos, 8,13 puntos, mientras que el promedio para la $\mathrm{C} 1$ fue de 6,96. Es decir que los y las estudiantes de la C2 lograron producir borradores que fueron superiores a las primeras construcciones de sus pares de la $\mathrm{C} 1$ en más de un punto. Sin embargo, esta diferencia de partida no redundó en lograr mejores niveles de desempeño en la versión editada.
Por otra parte, las Figuras 5 y 6 permiten observar el desempeño de los y las estudiantes en relación con las distintas dimensiones del género, comparando la versión borrador y la versión final.

En este caso, se evidencia que sin bien en ambas comisiones se produjo un avance en el desempeño de los y las estudiantes - en todos los casos, mejoraron la puntuación obtenida-, si se compara el rendimiento entre comisiones este progreso se

\section{Comparación entre ambas versiones de la descripción de flujograma en la C1}

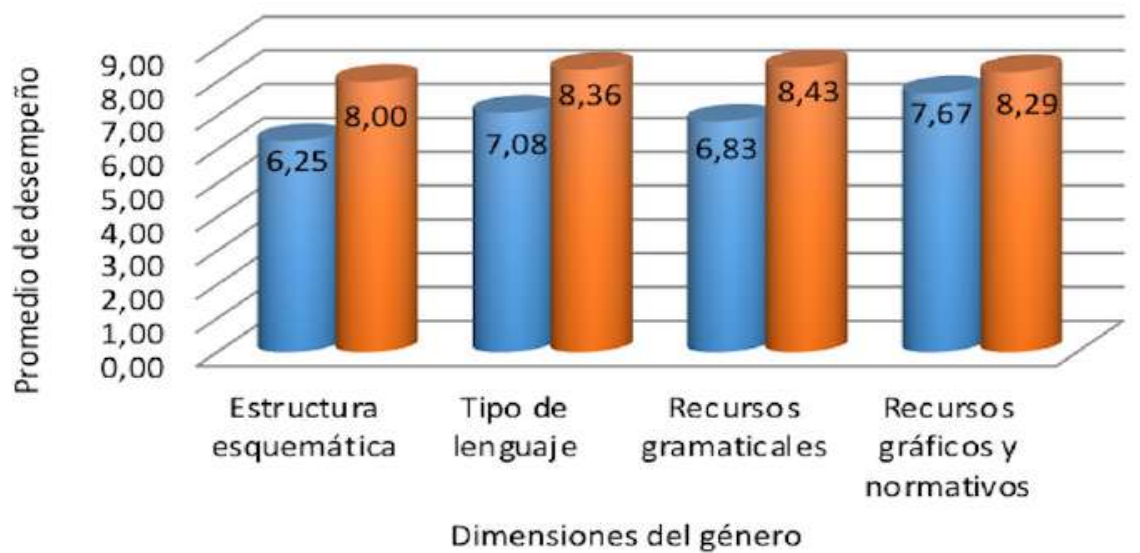

Barrad or

a Final

Figura 5 Comparación entre ambas versiones de la descripción de flujograma en la C1

\section{Comparación entre ambas versiones de la descripción de flujograma en la C2}

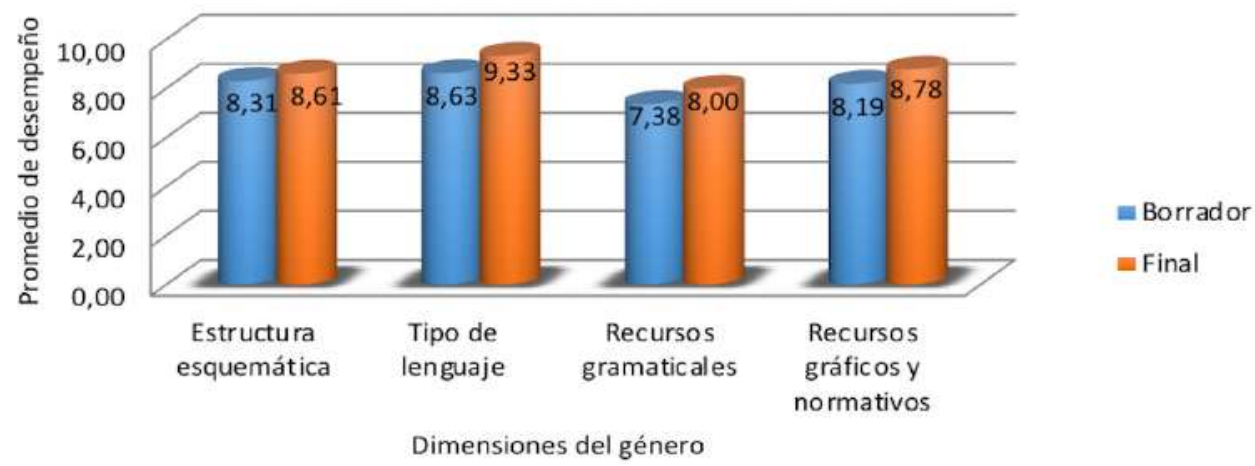

Figura 6 Comparación entre ambas versiones de la descripción de flujograma en la C2 
distribuye de forma diferenciada en cada grupo. Por ejemplo, mientras que la C1 avanzó notablemente en relación con la estructura esquemática (1,75 puntos), la C2 registró un menor nivel de progreso en esa dimensión (0,30 puntos). El mayor grado de avance en la $\mathrm{C} 2$, por su parte, se registra para la dimensión "Tipo de lenguaje" $(0,69)$, mientras que la misma dimensión es la segunda más baja para la $\mathrm{C} 1(1,27)$. Si se tiene en cuenta que en ambas comisiones se hicieron las mismas intervenciones con los mismos recursos, la disparidad en el rendimiento de los grupos debería explicarse en función de otras variables.

Al margen de este comentario, esto es, de la falta de sistematicidad en el avance relativo de cada dimensión para cada grupo, cabe mencionar que los sujetos de la $\mathrm{C} 1$ mostraron un progreso menos homogéneo entre los distintos aspectos del género, mientras que los de la C2 parecen estar atendiendo igualmente a todas las variables, por lo que logran un crecimiento menor, pero más equivalente entre dimensiones, como muestra la Figura 7. Para su lectura, hay que tener en cuenta que 1) representa la estructura esquemática; 2) el tipo de lenguaje; 3) los recursos gramaticales; y 4) los recursos gráficos y normativos. En este último caso, los valores correspondientes a las C1 y C2 están prácticamente superpuestos.

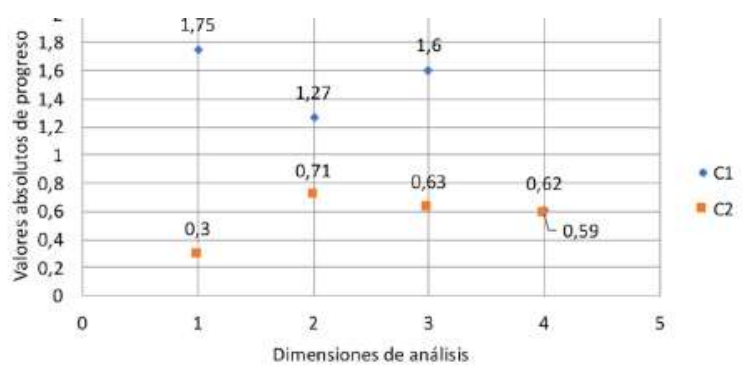

Figura 7 Dispersión de los valores absolutos de progreso de la $\mathrm{C} 1$ y la C2 entre la versión borrador y la versión final, según dimensión de análisis. 1: estructura esquemática; 2: tipo de lenguaje; 3: recursos gramaticales; 4: recursos gráficos y normativos.

\section{Discusión}

Los resultados obtenidos permiten una doble reflexión que aparece entramada en términos de la descripción genérica y en términos didácticos.

Ante todo, es posible afirmar que el rendimiento en la versión borrador o en la primera construcción de los escritos da cuenta de un importante trabajo previo, en el marco de la deconstrucción conjunta del género, y en relación con la subetapa diseño.

Resultados de entre 7 y 8 puntos en la elaboración del borrador de un macrogénero especializado por parte de estudiantes ingresantes al nivel superior constituyen un desempeño que merece ser observado. Esto es así no solo por la complejidad estructural, discursiva y léxico-gramatical del texto -que ha quedado al descubierto en la descripción presentada en este trabajo-, sino también por el desafío cognitivo que representa para quienes se enfrentan por primera vez con las exigencias del nivel superior y con los contenidos del campo disciplinar.

Estos datos resultan aún más contundentes si se tiene en cuenta que, según el "Censo nacional de población, hogares y viviendas 2010”, el $95 \%$ de la población ocupada del partido de Almirante Brown trabaja en relación de dependencia, en calidad de obreros o empleados, y que la mayor parte de la matrícula de la $\mathrm{UNaB}$ es primera generación que accede a la educación superior (Instituto Nacional de Estadística y Censos, 2012).

En este sentido, es posible suponer que el trabajo de deconstrucción conjunta del macrogénero seguido de la subetapa diseño, que permite abrir un espacio y un tiempo para discutir qué información contendrá el texto y en qué orden y por qué se presentará la información de ese modo- constituye una estrategia didáctica potente; es decir, los resultados de este trabajo pueden ser interpretados como una evidencia a favor del modelo didáctico utilizado.

Se debe recordar que, durante la deconstrucción, los y las estudiantes observan, analizan y 
sistematizan las características de un género o macrogénero con el andamiaje del docente (Martin, 1999; Rose y Martin, 2012; Wood et al., 1976), tomando como modelo una instanciación del género que constituye un ejemplar textual representativo. En este caso específico, luego de una lectura completa en voz alta, se discutió la macroestructura del texto y el propósito de cada etapa —es decir, de cada género dentro del macrogénero-.

En esta instancia, los y las estudiantes compartieron sus interpretaciones antes de sistematizar la información obtenida; luego, se releyeron las etapas del macrogénero, es decir, se enfocó en cada género, y se señalaron y comentaron sus recursos lingüísticos, siempre estableciendo conexiones entre esos recursos y el propósito global del macrogénero. Después, sobre la base de la información sistematizada, los y las estudiantes comenzaron a diseñar un texto de forma conjunta y tomaron nota para sus propios diseños.

En síntesis, es posible arriesgar que los buenos desempeños observados en promedio para la primera versión del texto se deban — al menos en parteal fuerte trabajo previo, al conocimiento profundo de un texto modelo y a la discusión en torno a sus características. En otras palabras, la tarea de lectura intensa y guiada podría haber operado favorablemente a la hora de escribir, y podría explicar el hecho de que ninguno de los trabajos escritos se haya alejado de los objetivos propuestos.

Reflexiones similares merece el análisis de los resultados que surgen de la comparación entre el desempeño en la primera construcción y en la segunda entrega, luego de la clase de edición. Ante todo, es importante recordar que los y las estudiantes no recibieron una devolución personalizada de sus producciones, por lo que el avance entre la primera instancia de escritura y la reelaboración representa aquello que los y las estudiantes pueden realizar por sí mismos, extrapolando al propio texto las reflexiones que llevaron a cabo a partir de un trabajo ajeno y en el marco de la discusión conjunta. Es decir, en ningún caso se señaló dónde y cómo reformular cada trabajo. Esta tarea la realizaron los sujetos por sí mismos luego de la clase grupal de discusión. Por lo tanto, es posible suponer que la tarea de modelaje de la actividad que realiza el docente durante la etapa edición, a través de lo que Rogoff (1994) llama "mente compartida", constituya una estrategia potente para dotar a los y las estudiantes de un mayor grado de consciencia respecto de su propia escritura. Cabe recordar que, en esta instancia, se modelan las "discusiones" que el autor tiene consigo mismo en el momento de producir y reelaborar su propio texto de manera autónoma. Esta clase de estrategias, por ende, permite simultáneamente caracterizar un género o macrogénero - en tanto quien guía la tarea se enfoca en sus elementos lingüísticos clave, característicos- y poner en escena el proceso de escritura.

En relación con todo esto, es decir, la reflexión sobre las etapas deconstrucción y edición y la subetapa diseño como parte del modelo didáctico seleccionado, cabe señalar que la apelación a la LSF como marco teórico constituye una potente herramienta didáctica. En efecto, no solo permite una caracterización minuciosa del género o macrogénero en la fase de planificación docente, sino que, además, habilita un metalenguaje común durante los distintos encuentros, lo que facilita la objetivación de la escritura y de su proceso de elaboración y, por lo tanto, la discusión en torno a estos temas.

Finalmente, en relación con la mayor o menor distancia entre ambas producciones si se compara el desempeño de las dos comisiones, es posible suponer que la disparidad entre los grupos dé cuenta de algunos de los fenómenos que inciden en el proceso de edición y de los tiempos propios del aprendizaje. Por ejemplo, los sujetos de la $\mathrm{C} 1$ asistieron en su totalidad a las intervenciones didácticas y todos ellos, salvo en un caso, produjeron ambas versiones. En el momento de edición conjunta, compartieron las dificultades enfrentadas durante la construcción individual y ensayaron caminos alternativos para resolver los problemas identificados. Por el contrario, en la C2, los sujetos presentaron un mayor nivel de ausentismo, 
por lo que muchos de ellos no participaron de los debates en el aula: se limitaron a utilizar el material disponible.

Además, durante la edición conjunta con la C2 se identificaron algunas dificultades particularmente desafiantes. En primer lugar, ante la necesidad de revisar las definiciones construidas en sus textos —en la etapa Clasificación-, los sujetos expresaron que les resultaba muy complejo identificar los elementos clave o centrales de la definición, que den cuenta exactamente de la identidad de la entidad que estaba siendo definida, el flujograma. En esta instancia, se generó un acalorado debate disciplinar, del que participaron docentes y estudiantes, y que los llevó a revisar los contenidos conceptuales abordados previamente. Sin embargo, en la mayor parte de los trabajos no se evidenció el impacto de este debate: las definiciones no lograron mayor precisión, lo que permite suponer que resulta necesario trabajar de forma más sostenida en la asignatura, para lograr un aprendizaje más profundo del contenido, o que debería haber habido una nueva instancia de edición, para seguir avanzando en este tema.

En segundo lugar, otro escollo se presentó al analizar la etapa Explicación, en relación específicamente con la construcción de una explicación que incluye secuencias optativas dependientes de elementos que introducen condicionalidad. En particular, los y las estudiantes debatieron largamente sobre cómo editar fragmentos textuales en los que no se lograban explicar de forma ordenada y clara las secciones del flujograma que incluían el bloque Toma de Decisión y Ramificación. En las versiones finales se evidenció una organización más ordenada y completa de las cláusulas y un mayor uso de conjunciones y metáforas gramaticales lógicas. Sin embargo, el trabajo con estos fragmentos hubiera requerido más tiempo, para lograr mejores resultados.

Por último, una tercera dificultad observada en el grupo se vincula con la tarea de escritura realizada antes por los mismos sujetos. En efecto, los y las estudiantes habían realizado previamente, en la misma asignatura y con los mismos docentes, la escritura de un manual de usuario (Serpa, 2019) y muchas de las características de este género fueron trasladadas directamente al macrogénero DF. Por ejemplo, algunos sujetos forzaban el texto para incluir al lector en figuras directivas típicas de los géneros instructivos o explicaban el proceso del flujograma en términos de pasos a seguir. Estos temas fueron debatidos durante la edición conjunta, pero no fueron aprehendidos por todos los sujetos.

Los tres puntos mencionados constituyen algunas líneas de análisis cualitativo para buscar dar cuenta del menor grado de avance de los sujetos de la $\mathrm{C} 2$ respecto de los de la $\mathrm{C} 1$ entre las distintas versiones producidas a lo largo del proceso. Dos de estas líneas - el problema de las definiciones y el problema de las explicaciones secuenciales condicionales - conducen directamente a reflexionar sobre la potencia de discutir la escritura en el marco de las asignaturas, dado que apuntan a su carácter de herramienta epistémica (Bereiter y Scardamalia, 1987; Halliday, 1993; Vigotsky, 1988, 1998). En cualquier caso, y más allá de que se trata de meras hipótesis explicativas, cabe señalar que instancias futuras de trabajo con el mismo género podrían atender a tales "nodos problemáticos", a fin de lograr mejores desempeños. ${ }^{3}$ No obstante, no se debe perder de vista que, en todos los casos, el nivel de desempeño entre borrador y versión final fue mayor.

Por otra parte, es posible enriquecer estos resultados ampliando el ángulo de interpretación. En efecto, si se tiene en cuenta que, en el mismo semestre, los y las estudiantes de este grupo realizaron previamente otro trabajo de escritura bajo el mismo modelo didáctico (Serpa, 2019), los datos cobran un matiz especial en su apreciación

3 Durante el segundo semestre de 2020, un nuevo grupo de estudiantes trabajó en la asignatura para la producción del mismo macrogénero, acompañados por la misma docente. Se espera poder evaluar los resultados de esta nueva tarea y compararlos con los que se discuten aquí. 


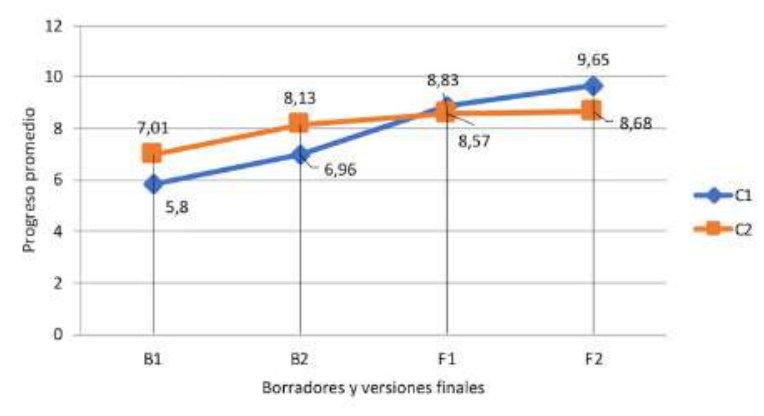

Figura 8 Progreso promedio durante el segundo semestre de 2019, según comisiones

longitudinal. La Figura 8 ilustra el avance de ambos grupos durante el semestre, dado que representa el progreso en el tiempo entre borradores (B1 para $\mathrm{C} 1$, y B2 para $\mathrm{C} 2$ ) y versiones finales (F1 y F2, respectivamente).

Como se puede observar, los grupos muestran una tendencia creciente sostenida. Si a este dato se suma una comparación respecto de las dimensiones del texto a las que han podido atender los sujetos en la escritura del primer macrogénero y el segundo, el crecimiento es aún mayor. En efecto, al trabajar con el primero, el manual de usuario, los sujetos colocaban mayor énfasis en una u otra dimensión del texto. Mientras que en este segundo macrogénero, la $\mathrm{DF}$, el progreso es más homogéneo en todas las dimensiones (véanse las Figuras 5 y 6 ) (Serpa, 2019).

En relación con todo lo anterior, entonces, es posible ubicar este trabajo en el marco de los debates en torno a la enseñanza de la escritura en la educación superior y su didáctica específica, sin perder de vista la problemática de la inserción de esta enseñanza en el nivel y la relación entre escritura y aprendizaje. Los resultados respaldan, al menos provisoriamente, la tarea que lleva a cabo la Subárea Competencias en Discurso Profesional y Académico de la $\mathrm{UNaB}$, y refuerzan el presupuesto de que los géneros de la educación superior presentan ciertas especificidades propias de los campos disciplinares y de las demandas del ámbito universitario, que resultan novedosas para los y las estudiantes, y que reclaman, por tanto, ser enseñadas y aprendidas de la mano del resto de los contenidos. En este sentido, tanto la descripción de géneros y macrogéneros (Eggins y Martin, 2003; Martin, 1992, 1994, 2001, 2014; Martin y Rose, 2008), como la enseñanza en las asignaturas y a lo largo de las carreras (Moyano, 2007, 2009, 2010, 2013, 2017, 2018; Moyano y Giudice, 2016) parecen constituir una opción adecuada para ello.

En este sentido, el desempeño de las y los estudiantes de HCyP durante el segundo semestre de 2019 constituye una primera prueba a favor de la propuesta institucional de la $\mathrm{UNaB}$ y, en particular, del encuadre didáctico de la Subárea, en su pretensión de superar la "inclusión excluyente" que denuncia Ana María Ezcurra (2011). Se espera que el seguimiento de los sujetos a lo largo de sus carreras, durante los próximos semestres, termine por demostrar si la apuesta por esta forma de trabajo orientada a asegurar la permanencia y la graduación de los sujetos resulta tan satisfactoria como augura en sus inicios.

\section{Referencias}

Bereiter, C. y Scardamalia, M. (1987). The psychology of written composition. Erlbaum.

Bernstein, B. (1990). Class, codes and control. Vol 4. The structuring of Pedagogic Discourse. Routledge.

Eggins, S. (2004). An introduction to systemic functional linguistics. Continuum.

Eggins, S. y Martin, J. (2003). El contexto como género: una perspectiva lingüístico-funcional. Revista Signos, 36(54), 185-205. https://doi.org/10.4067/ S0718-09342003005400005

Ezcurra, A. M. (2011). Igualdad en educación superior. Un desafío mundial. Universidad Nacional de General Sarmiento.

Halliday, M. (1993). Towards a language-based theory of learning. Linguistics and Education, 5(2), 93-116. https://doi.org/10.1016/0898-5898(93)90026-7

Halliday, M. (2004). The language of sciences. Continuum.

Halliday, M.yC. Matthiessen (2004). Clause as representation. En Halliday, M. y C. Matthiessen. An introduction to funtional grammar (pp. 168-305). Arnold. 
Hood, S. y Martin, J. (2005). Invocación de actitudes: el juego de la gradación de la valoración en el discurso. Revista Signos, 38(58), 195-220. https://doi. org/10.4067/S0718-09342005000200004

Hyland, K. (2002). Teaching and researching writing. Longman.

Instituto Nacional de Estadística y Censos. (2012). Censo nacional de población, hogares y viviendas 2010: Censo del Bicentenario. Resultados definitivos, Serie $B n^{\circ}$ 2. Instituto Nacional de Estadística y Censos.

Martin, J. (1992). English text. System and structure. Benjamins. https://doi.org/10.1075/z.59

Martin, J. (1994). Macro-genres: The ecology of the page. Network 21(1), 29-52.

Martin, J. (1999). Mentoring semogenesis: 'Genre-based' literacy pedagogy. En F. Christie (Ed.), Pedagogy and the Shaping of Consciousness. Linguistic and Social Processes (pp. 123-155). London: Cassell.

Martin, J. (2000a). Beyond exchange: Appraisal systems in English. En S. Hunston y G. Thompson (Eds.), Evaluation in text. Authorial stance and the construction of discourse (pp. 142-175). Oxford University Press.

Martin, J. (2000b). Factoring out exchange: types of structure. En M. Coulthard, J. Cotterill y F. Rock (Eds.), Working with Dialogue (pp. 19-40). Niemeyer.

Martin, J. (2001). A context for genre: Modelling social processes in functional linguistics. En J. Devilliers y R. Stainton (Eds.), Communication in linguistics. Papers in honour of Michael Gregory (pp. 287-328). Gref Publishers.

Martin, J. (2014). Evolving systemic functional linguistics: Beyond the clause. Functional Linguistics, 1(3): 2-24. https://doi.org/10.1186/2196-419X-1-3

Martin, J. R. y White, P. (2005). The language of evaluation. Appraisal in English. Palgrave. https://doi. org/10.1057/9780230511910

Martin, J. y Rose, D. (2007). Working with discourse. Meaning beyond the clause. Bloomsbury.

Martin, J. y Rose, D. (2008). Genre relations. Mapping culture. Equinox.

Moyano, E. (2004). La escritura académica: una tarea interdisciplinaria a lo largo de la curricula universitaria. Revista Texturas, 4(4), 109-120. https://doi. org/10.14409/texturas.v1i4.2824

Moyano, E. (2007). Enseñanza de habilidades discursivas en español en contexto pre-universitario: una aproximación desde la LSF. Revista Signos,
40(65), 573-608. https://doi.org/10.4067/ S0718-09342007000300009

Moyano, E. (2009). Negotiating genre: Lecturer's awareness in genre across the curriculum project at the university level. En Ch. Bazerman, A. Bonini y D. Figueiredo (Eds.), Genre in a changing world. Perspectives on writing (pp. 449-464). The WAC Clearinghouse and Parlor Press.

Moyano, E. (2010). Escritura académica a lo largo de la carrera: un programa institucional. Revista Signos, 43(74), 465-488. https://doi.org/10.4067/ S0718-09342010000500004

Moyano, E. (2011). Deconstrucción y edición conjuntas en la enseñanza de la escritura [Ponencia]. Anais vi Simpósio Internacional de Estudios de Gêneros Textuais (VI SIGET). Universidade Federal do Rio Grande do Norte.

Moyano, E. (2013). Una didáctica de las ciencias basada en los géneros textuales: acceso a las disciplinas a través de la apropiación de su discurso. En: E. I. Moyano (Coord.), Aprender ciencias y humanidades: una cuestión de lectura y escritura. Aportes para la construcción de un programa de inclusión social a través de la educación lingüistica (pp. 109-155). Universidad Nacional de General Sarmiento.

Moyano, E. (2017). Diseño e implementación de programas de lectura y escritura en el nivel universitario: principios y estrategias. Revista Lenguas Modernas, 50, 47-72.

Moyano, E. (2018). La enseñanza de la lectura y la escritura académicas mediante un programa a lo largo del curriculum universitario: opción teórica, didáctica y de gestión. DELTA, 34(1): 235-267. https://doi. org/10.1590/0102-445074896274115057

Moyano, E. y Giudice, J. (2016). Negotiation between professional peers: Critical strategy for a reading and writing program at the university level. Ilha do Desterro, 69(3), 157-172. https://doi. org/10.5007/2175-8026.2016v69n3p157

Norton, P. y Wiburg, K. (2003). Teaching with technology. Designing opportunities to learn. Wadsworth/Thomson Learning.

Rogoff, B. (1994). Los tres planos de la actividad sociocultural: apropiación participativa, participación guiada y aprendizaje. En J. V. Wertsch., P. del Río y A. Álvarez (Eds.), La mente sociocultural. Aproximaciones teóricas y aplicadas (pp. 111-128). Fundación Infancia y Aprendizaje.

Rose, D. y Martin, J. R. (2012). Learning to write, reading to learn. Genre, knowledge and pedagogy in the Sydney School. Equinox. 
Serpa, C. (2019). El manual de usuario en el campo de las herramientas computacionales: el género y su enseñanza [Manuscrito no publicado]. Subárea en Discurso Profesional y Académico, UNaB.

Universidad Nacional Guillermo Brown (UNaB). (s. f.). Automatización. https://www.unab.edu.ar/automatizacion.html
Vigotsky, L. (1988). El desarrollo de los procesos psicológicos superiores. Crítica Grijalbo.

Vigotsky, L. (1998). Pensamiento y lenguaje. Fausto.

Wood, D., Bruner, J. y Ross, G. (1976). The role of tutoring in problem solving. Journal of Child Psychology and Psychiatry, 17(2), 89-100. https://doi. org/10.1111/j.1469-7610.1976.tb00381.x

Cómo citar este artículo: Serpa, C. (2021). La enseñanza de la escritura en la educación superior: una experiencia didáctica en torno al macrogénero "Descripción de Flujograma". Íkala, Revista de Lenguaje y Cultura, 26(1), 77-96. https://doi.org/10.17533/udea.ikala.v26n01a02 